\title{
Steckt hinter jeder Reizblase eine Infektion?
}

n den aktuellen Leitlinien der AmeriJahr 2014 ist das „overactive bladder syndrom“ (OAB) definiert als „Harndrang meist kombiniert mit hoher Miktionsfrequenz und Nykturie mit und ohne Dranginkontinenz, dem weder ein Harnwegsinfekt noch andere offensichtliche Pathologien zugrunde liegen dürfen“. Damit sei schon definitionsgemäß eine Harnwegsinfektion als OAB-Ursache ausgeschlossen, erklärte Dr. Zafer Tandoğdu vom Northern Institute for Cancer Research der Universität in Newcastle Upon Tyne. Doch die äußerst unterschiedlichen Angaben zur OAB-Prävalenz, die von $9-42 \%$ reichen, werfen Frage auf und zwingen zur genaueren Betrachtung, so Tandoğdu.

Sofort ins Auge fallen die zahlreichen Überschneidungen von $\mathrm{OAB}$ und Harnwegsinfektion (HWI), wie Tandoğdu betonte. So seien in beiden Fällen überwiegend Frauen betroffen, und die Er- krankungszahlen steigen mit zunehmendem Alter. Zudem seien bei OAB wie auch HWI die zugrunde liegenden Pathomechanismen komplex und noch nicht vollständig geklärt. Bei OAB etwa werden neben einer Schädigung des Urothels auch neurogene und muskuläre Veränderungen als Ursachen diskutiert. Bei HWI sind ebenfalls die urothelialen Zellen betroffen. Dabei siedeln viele Bakterien, wie sich mittlerweile herausgestellt habe, nicht nur außen auf dem Blasenepithel an, sondern können sich in die Zellen einnisten und intrazelluläre Kolonien bilden. Damit stehe die Aussagekraft der gängigen Diagnoseverfahren infrage, erklärte Tandoğdu und verwies auf die aktuelle Mikrobiomforschung. Verglichen mit den neuen molekularbiologischen Analyseverfahren bleiben bei den Standardmethoden in $92 \%$ der Fälle Bakterien unentdeckt, so Tandoğdu. Tatsächlich fanden sich bei Frauen ohne HWI-Symp- tome und mit negativer Bakterienkultur häufiger intrazelluläre Bakterienkolonien, wenn sie über Beschwerden des unteren Harntrakts (LUTS) berichtet hatten im Vergleich zu Beschwerdefreien ( $94 \%$ vs. $2 \%$ ). Außerdem differierten die Mikrobiota in beiden Gruppen deutlich.

Offensichtlich seien die gängigen mikrobiellen Diagnosemethoden nicht sensitiv genug, um HWI sicher zu diagnostizieren, fasste Tandoğdu zusammen. Gleichzeitig finden sich bei Patienten mit OAB-Symptomen häufiger intrazelluläre Bakterienkolonien sowie vermutlich eine differierende Mikrobiomzusammensetzung. Womöglich bleiben bei vielen OAB-Patienten HWI schlichtweg unentdeckt, spekulierte der Urologe. Dennoch warnte er eindringlich davor, daraus schlussfolgern zu wollen, Antibiotika wären eine geeignete Therapieoption für das Krankeitsbild überaktive Blase.

Dr. Dagmar Kraus

"Is OAB an UTI?", Z. Tandoğdu, EAU-Kongress, München, 12. März 2016

\section{Das Mikrobiom und der Mythos vom sterilen Urin}

W enn klassische mikrobielle Methoden im Urin keine Mikroorganismen anzeigen, heißt das nicht, dass dort keine sind, erklärte Dr. Vitaly Smelov vom Karolinska-Institut in Schweden. Vielmehr tummeln sich in den Harnwegen offenbar auch bei Gesunden eine ganze Menge von Mikroben, wie Untersuchungen mit neuen molekularbiologischen Analysemethoden ergeben haben. Die Gesamtheit aller vorkommenden $\mathrm{Mi}$ kroorganismen bezeichnet man als $\mathrm{Mi}-$ krobiota, deren kollektive Genome als Mikrobiom. Das Mikrobiom sei aber keine statische Sache. Zwar kommen bestimmte Mikroorganismen wohl in jedem Menschen vor, deren Genome bilden das sogenannte Kernmikrobiom, es existiere aber auch ein variabler Teil, der durch verschiedene Faktoren wie zum Beispiel Lebensstil, Immunsystem, Medikamen- teneinnahme oder Krankheiten beeinflusst wird. Aus der Forschung am Darm weiß man, dass das Mikrobiom je nach Zusammensetzung chronische Entzündungen verursachen, Gene und somit den Metabolismus menschlicher Zellen aktivieren sowie die Immunantwort auf Krebszellen beeinflussen kann. Beim Urogenitaltrakt stehe die Forschung zwar noch am Anfang, doch zeigten erste Studien, dass sich beispielsweise die mikrobiellen Populationen in Urin, Samenflüssigkeit und Prostatasekret bei Männern mit benigner Prostatahyperplasie und Männern mit Prostatakarzinom signifikant unterscheiden. Smelov hofft auf lange Sicht gesehen, mit Erforschung des Mikrobioms im Urogenitaltrakt mehr über dessen Bedeutung bei der Krankheitsentstehung zu erfahren.

Der Labormediziner warnte allerdings eindringlich davor, mit der Implementierung sensi- tiverer Nachweismethoden die Antibiotikaverschreibung mit all ihren Nebenwirkungen und Kollateralschäden zu forcieren. Denn der „sterile“ Urin sei wie die neueren Analysemethoden zeigen - ein Mythos, und eine Bakteriurie beweise nicht zwingend ein infektiöses Geschehen. Vielmehr stehe sie für eine Kolonisation oder könne als Risikofaktor gelten. Sie indiziere aber nicht grundsätzlich eine antibiotische Behandlung. Vielmehr könne eine bestimmte Kolonisation sogar vor wiederkehrenden Infekten schützen, erklärte Smelov und nannte als praktisches Beispiel die Instillation von Escherichia coli in die Blase bei rezidivierender Zystitis. Smelov hofft, gerade vor dem Hintergrund der rasant wachsenden Antibiotikaresistenzen, mit den neuen sensitiveren Methoden die wahren Uropathogene identifizieren und somit die antibakterielle Therapie optimieren zu können.

Dr. Dagmar Kraus

"The role of the microbiome in the development of urinary tract problems", V. Smelov, EAU-Kongress, München, 12. März 2016 